\title{
Identification of drought-responsive microRNAs in Medicago truncatula by genome-wide high- throughput sequencing
}

Tianzuo Wang ${ }^{1,2}$, Lei Chen ${ }^{1,2}$, Mingui Zhao ${ }^{1}$, Qiuying Tian ${ }^{1}$ and Wen-Hao Zhang ${ }^{\text {** }}$

\begin{abstract}
Background: MicroRNAs (miRNAs) are small, endogenous RNAs that play important regulatory roles in development and stress response in plants by negatively affecting gene expression post-transcriptionally. Identification of miRNAs at the global genome-level by high-throughout sequencing is essential to functionally characterize miRNAs in plants. Drought is one of the common environmental stresses limiting plant growth and development. To understand the role of miRNAs in response of plants to drought stress, drought-responsive miRNAs were identified by high-throughput sequencing in a legume model plant, Medicago truncatula.

Results: Two hundreds eighty three and 293 known miRNAs were identified from the control and drought stress libraries, respectively. In addition, 238 potential candidate miRNAs were identified, and among them 14 new miRNAs and 15 new members of known miRNA families whose complementary miRNA*s were also detected. Both high-throughput sequencing and RT-qPCR confirmed that 22 members of 4 miRNA families were up-regulated and 10 members of 6 miRNA families were down-regulated in response to drought stress. Among the 29 new miRNAs/ new members of known miRNA families, 8 miRNAs were responsive to drought stress with both 4 miRNAs being up- and down-regulated, respectively. The known and predicted targets of the drought-responsive miRNAs were found to be involved in diverse cellular processes in plants, including development, transcription, protein degradation, detoxification, nutrient status and cross adaptation.

Conclusions: We identified 32 known members of 10 miRNA families and 8 new miRNAs/new members of known miRNA families that were responsive to drought stress by high-throughput sequencing of small RNAs from $M$. truncatula. These findings are of importance for our understanding of the roles played by miRNAs in response of plants to abiotic stress in general and drought stress in particular.
\end{abstract}

\section{Background}

Endogenous small interfering RNAs (siRNAs) and microRNAs (miRNAs) are the two most abundant classes of plant small RNAs (sRNAs). The small RNAs are processed in the nucleus from longer precursor transcripts that form distinct secondary structures. The miRNAs down-regulate gene expression by targeting specific messenger RNAs (mRNAs) in both plants and animals $[1,2]$.

miRNAs were initially discovered in Caenorhabditis elegans as developmental timing regulators in 1994 [3].

\footnotetext{
* Correspondence: whzhang@ibcas.ac.cn

'State Key Laboratory of Vegetation and Environmental Change, Institute of Botany, the Chinese Academy of Sciences, Beijing 100093, PR China Full list of author information is available at the end of the article
}

The existence of miRNAs in organisms including plants, mammals and virus has widely been recognized. The biogenesis of miRNAs in plants is a multi-step enzymatic process involving incorporation of miRNAs into the RNA-induced silencing complex (RISC), and then miRNAs bind target mRNAs to direct for cleavage miRNAs with near perfect complementarity and/or inhibiting translation of those with lower complementarity [4-7]. There has been ample evidence demonstrating that miRNAs play a regulatory role in diverse biochemical and physiological processes in plants $[1,4]$. For instance, miRNAs have been shown to play a role in the modulation of the processes associated with growth and development in plants, including leaf morphogenesis, floral organ and root development [6,8-13]. In addition, 
recent studies also revealed that miRNAs are involved in responses of plants to various abiotic and biotic stresses. These include drought [14-18], cold [19-21], salinity [22], nutrient starvation [23-26], oxidative stress [27], submergence [28], UV-B radiation [29,30] and virus [31,32].

Plants are hardly grown under optimal conditions, and have to frequently suffer from drought stress due to shortage of water supply. Not unexpectedly, the involvement of miRNAs in response of plants to drought has been evaluated in several plant species. A number of miRNAs have been identified to be associated with response to drought stress in several species such as Arabidopsis thaliana $[14,33]$ and Oryza sativa $[17,18]$. For instance, Zhou et al. (2010) identified 11 down-regulated miRNAs and 8 upregulated miRNAs in response of rice to drought [18]. It has been reported that miR $169 \mathrm{~g}$ is the only member induced by drought in the miR169 family of rice and its expression is regulated by drought [17]. Li et al. (2008) found that miR169a and miR169c are substantially downregulated by drought, leading to the enhanced resistance to drought in Arabidopsis because one of the miR169's targets, NFYA5 (Nuclear Factor YA5), is a crucial transcription factor regulating the expression of a number of drought stress-responsive genes [14]. Recent studies also demonstrated that miRNAs play a role in control of drought resistance in maize by modulating the expression of MAPK (mitogen-activated protein kinase), PLD (phospholipase D), PDH (praline dehydrogenase) and POD (peroxidase) [16]. More recently, Trinadade et al. (2010) reported that miR398 and miR408 are up-regulated by water deficit in Medicago truncatula, leading to a downregulation of their target genes of $C O X 5 b, C S D 1$ and plantacyanin [15]. In Triticum dicoccoides, 13 miRNAs are differentially regulated in response to short-term drought stress by miRNA microarray chip [34]. Despite of numerous studies on the involvement of miRNAs in abiotic stress in general and drought stress in particular, there has been no report on the systemic identification of droughtresponsive miRNAs in leguminous plants at the global genome-level by high-throughout sequencing.

Leguminous plants account for one third of primary crop production in the world and are important sources for the consumption of human and animals [35]. Drought stress is one of the most frequently occurred environmental stresses that limit crop yield world-wide. Medicago truncatula is an annual legume species distinguished by its small diploid genome and easy transformation, and is a model plant to study functional genomics of legume plants [36]. Although numerous miRNAs have been identified in various plant species and the mechanisms underlying their actions are being unravelled, the discovery of new miRNAs in plants on a genome-wide scale is essential for functional characterization of miRNAs. The traditional sequencing of relatively small-size cDNA libraries of plant sRNAs from Arabidopsis, rice and poplar with Sanger method has led to the conclusions that plant miRNAs are highly conserved [37]. However, a small number of miRNAs was not detected in the genomes of some species, indicating that miRNAs may evolve more recently [38]. In addition, the non-conserved miRNAs are often expressed at a lower level than the conserved miRNAs, thus many nonconserved miRNAs cannot be detected in small-scale sequencing studies. In this context, high-throughput sequencing has been used to identify non-conserved miRNAs in several species [20,39-53]. To understand the role of miRNAs in response of plants to drought stress, we identified a number of conserved and non-conserved miRNAs that were responsive to drought by high-throughput sequencing, and their potential role in drought tolerance was discussed.

\section{Results}

\section{High-throughput sequencing of small RNAs from} M. truncatula

Two sRNA libraries in which $M$. truncatula seedlings were treated with drought stress (DS) and without stress, control (CK) constructed from shoots of seedlings grown under the two treatment-regimes were sequenced by an IlluminaSolexa sequencer. High-throughput sequencing of CK and DS libraries led to generation of $6,808,877$ and $6,874,742$ primary reads, respectively. There were $6,420,234$ clean reads $(2,685,754$ unique sequences) for $\mathrm{CK}$ and 6,505,965 clean reads $(2,937,417$ unique sequences) for DS after initial processing (Table 1). The length distribution of reads showed that the majority of the reads was 20-25 nt in size, of which the class of 24 nt was the most abundant group followed by the group of 21 nt class (Figure 1a).

The common/specific sequences were analyzed between CK and DS libraries for the total and unique sequences. There were $19.14 \%$ and $44.95 \%$ specific sequences in the DS library for total and unique sequences, respectively.

After initial processing, the high-quality small RNA reads were mapped to the $M$. truncatula genome sequence (Mt3.0) using SOAP [54]. The number of total and unique sequences that matched genome was $4,875,034 / 1,713,480$ and $4,819,359 / 1,867,375$ in the two libraries, respectively (Table 1 ).

\section{Identification of known miRNAs}

The known miRNAs in $M$. truncatula were identified by comparing with the up-dated miRNAs database (miRBase 17, released in April 26, 2011) [55]. We identified 283 and 293 known miRNAs in the two libraries, respectively (Table 1 ). Sixty-six members belonging to 
Table 1 Statistics of sRNA sequences for control (CK) and drought stress (DS) libraries

\begin{tabular}{lcc}
\hline & Number of reads & Number of unique sequences \\
\hline Control (CK) & & \\
$\quad$ Primary reads & $6,808,877$ & $2,685,754$ \\
Clean reads & $6,420,234$ & $1,713,480$ \\
Sequences mapped to the genome & $4,875,034$ & 283 \\
match known miRNAs & 509,981 & $2,937,417$ \\
\hline Drought stress (DS) & & $1,867,375$ \\
Primary reads & $6,874,742$ & 293 \\
Clean reads & $6,505,965$ & $2,819,359$ \\
$\quad$ Sequences mapped to the genome & 588,990 & \\
$\quad$ Match known miRNAs & & \\
\hline
\end{tabular}

14 conserved miRNA families were obtained after removing those miRNAs whose expression levels are too low to be analysed for differential expression. Of these families, the most abundant two reads were miR156 and miR166. Among the identified miRNAs, miR399 contained the most members, including miR399a-i and miR399k-q (Figure 1b).

In addition to the conserved miRNAs, we also identified 137 non-conserved miRNAs that belong to 44 miRNA families (Additional file 1) after removing miRNAs with expression levels that were too low to be analysed for differential expression. Among the 137non-conserved miRNAs, some were legume-specific. The most abundant miRNA was miR2086 followed by miR2111 in the control (CK) library. In the drought stress (DS) library, miR2111 was the most abundant, followed by miR1507. In addition, miR2630 was found to have the most members in the two libraries (miR2630a-y).

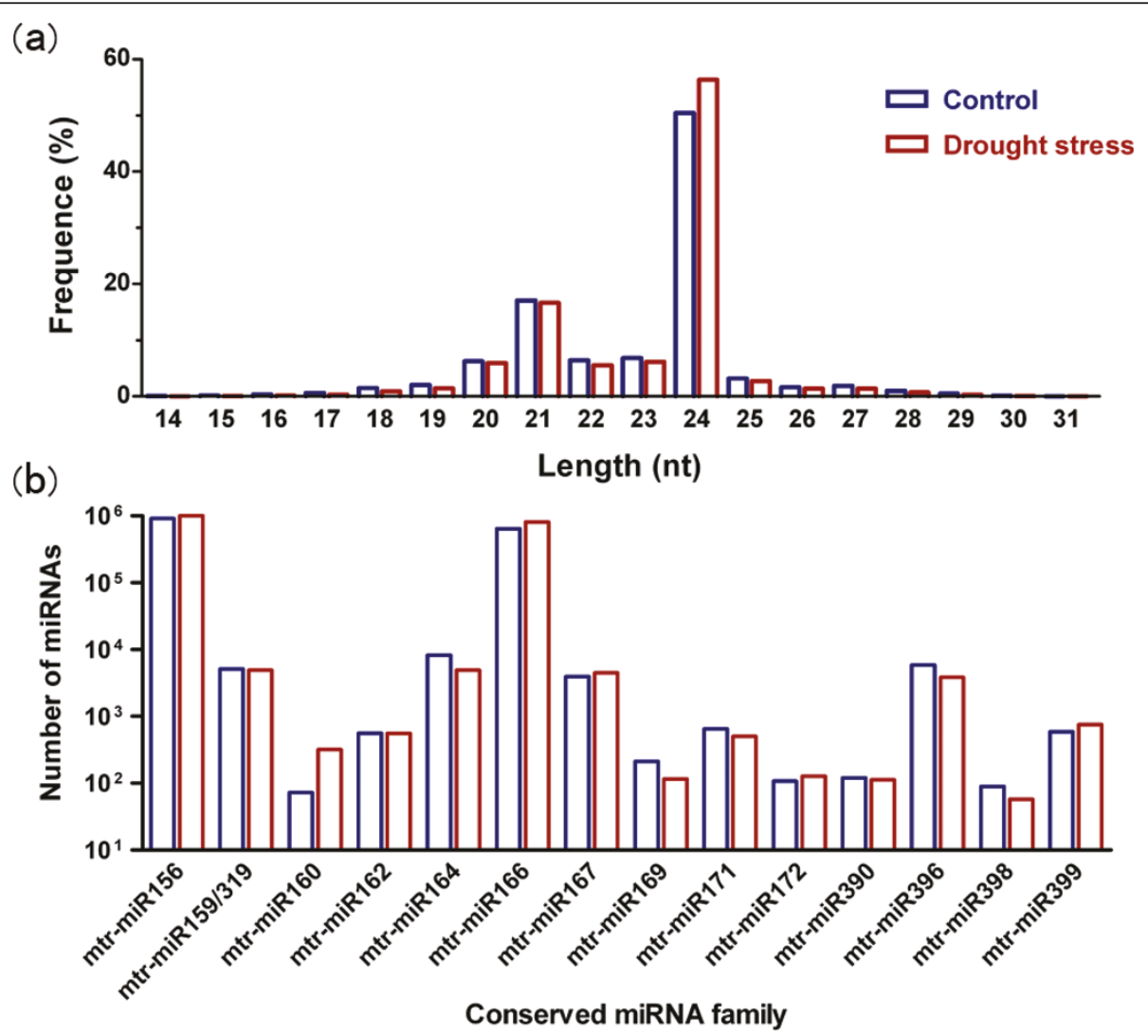

Figure 1 Distribution of small RNAs from control and drought stress libraries. The size distribution of small RNAs is shown in panel (a) with frequency. The absolute miRNA number in different conserved families is shown in panel (b). 


\section{Identification of new miRNAs/new members of known miRNA families}

The formation of stable hairpin structure is one of the essential features for identification of new miRNAs [56]. To identify new miRNAs and new members of known miRNA families, we pooled the reads obtained from the two libraries, and identified the miRNAs by folding the sequences of potential miRNA precursors using the mfold web server [57]. We obtained 238 potential miRNA candidates (Table 2, Additional file 2). In addition to the hairpin structure, detection of miRNA*s is another proof that has been widely used to confirm the new miRNAs [58]. Among the 238 potential miRNA candidates, we found 29 miRNAs with complementary
miRNA*s (Table 2, Figure 2, Additional file 3), indicating that these candidate miRNAs are likely to be new miRNAs or new members of known miRNA families in M. truncatula. The counts of several miRNA*s of these miRNAs were low (Table 2). This may make the annotation of these new miRNAs questionable. However, no requirement on the counts of several miRNA*s was given in the most recent criteria for annotation of plant miRNAs [58]. Therefore, these miRNAs were still considered to be new miRNAs or new members of known miRNAs. A similar identification of new miRNAs has also been reported by others in the literature. For instance, candidate miRNAs with only one miRNA* have been identified as new miRNAs in rice [59].

Table 2 New miRNAs and new members of known miRNA families with miRNA*s in $M$. truncatula

\begin{tabular}{|c|c|c|c|c|c|c|c|}
\hline miRNAs & Sequence $\left(5^{\prime}-3^{\prime}\right)$ & $\begin{array}{l}\text { Length } \\
\qquad(\mathrm{nt})\end{array}$ & $\begin{array}{l}\text { Counts of miRNAs/ } \\
\text { miRNA*s }\end{array}$ & Location & Arm & $\begin{array}{l}\text { Length of } \\
\text { precursors (nt) }\end{array}$ & $\begin{array}{l}\text { MFE (kcal/ } \\
\text { mol) }\end{array}$ \\
\hline miR5213 & uacgugugucuucaccucugaa & 22 & $15045 / 44$ & $\begin{array}{c}\text { MtChr2:16737666:16737796: } \\
+\end{array}$ & $5^{\prime}$ & 131 & -43.1 \\
\hline miR5274b & auaugacggaguguaaaugcc & 21 & $29 / 2$ & MtChr4:11424365:11424459:- & $5^{\prime}$ & 95 & -66.3 \\
\hline miR5554a & ugugcaucuugaacaaugguau & 22 & $134 / 3$ & MtChr1:31285750:31285854:- & $5^{\prime}$ & 105 & -52.8 \\
\hline miR5554b & ugugcaucuugaacaaugguau & 22 & $134 / 3$ & MtChr4:41013393:41013497:- & $5^{\prime}$ & 105 & -57.3 \\
\hline $\operatorname{miR5554c}$ & ugugcaucuugaacaaugguau & 22 & $134 / 1$ & MtChr7:19742691:19742795:- & $5^{\prime}$ & 105 & -53.3 \\
\hline miR5555 & uaagaguauaauaugacuuug & 21 & 40664 & MtChr1:12905862:12905956:- & $5^{\prime}$ & 95 & -47.5 \\
\hline $\operatorname{miR5556}$ & ugaugacggaagaaauccaaa & 21 & $155 / 1$ & MtChr4:23199944:23200064:- & $3^{\prime}$ & 121 & -54.8 \\
\hline miR5557 & ugcuuccuuaguacuuguuga & 21 & $5 / 3$ & MtChr5:6026167:6026481:+ & $3^{\prime}$ & 315 & -96.7 \\
\hline miR5558 & uuuuccaauucuaagucuauc & 21 & $139 / 8$ & $\begin{array}{c}\text { MtChr5:20521087:20521172: } \\
+\end{array}$ & $5^{\prime}$ & 86 & -33 \\
\hline miR5559 & uacuuggugaauuguuggauc & 21 & $2124 / 2$ & MtChr6:937306:937443:+ & $5^{\prime}$ & 138 & -48.8 \\
\hline $\operatorname{miR5} 560$ & ugccggcucaaugaaugcggag & 22 & $62 / 2$ & MtChr6:4747428:4747538:+ & $3^{\prime}$ & 111 & -53.8 \\
\hline $\operatorname{miR5561}$ & cauuuggagagacauagacaa & 21 & $449 / 3$ & MtChr7:30170572:30170660:- & $5^{\prime}$ & 89 & -42.9 \\
\hline $\operatorname{miR5} 562$ & uguggagucuuuugcaugaag & 21 & $16 / 1$ & MtChr7:32194444:32194667:- & $5^{\prime}$ & 224 & -56.4 \\
\hline $\operatorname{miR5563}$ & ugauaucaggcaacucggucc & 21 & $12 / 1$ & MtChr8:32699524:32699624:- & $5^{\prime}$ & 101 & -52.9 \\
\hline miR156j & uugacagaagagggugagcaca & 22 & $10341 / 23$ & MtChr1:3228429:3228554:+ & $5^{\prime}$ & 126 & -44.4 \\
\hline $\operatorname{miR} 167 b$ & ugaagcugccagcaugaucug & 21 & $40937 / 1$ & $\begin{array}{c}\text { MtChr7:34009590:34009796: } \\
+\end{array}$ & $5^{\prime}$ & 207 & -78.7 \\
\hline miR168c & ucgcuuggugcaggucgggaa & 21 & $56024 / 1434$ & $\begin{array}{c}\text { MtChr5:10397699:10397824: } \\
+\end{array}$ & $5^{\prime}$ & 126 & -68.8 \\
\hline $\operatorname{miR} 172 b$ & agaaucuugaugaugcugcau & 21 & $35344 / 63$ & AC235487:197944:198077:+ & $3^{\prime}$ & 134 & -55.5 \\
\hline $\operatorname{miR} 172 \mathrm{c}$ & agaaucuugaugaugcugcau & 21 & $35386 / 8$ & AC233663:10168:10308:+ & $3^{\prime}$ & 141 & -52.8 \\
\hline miR408 & augcacugccucuucccuggc & 21 & $75 / 55$ & $\begin{array}{c}\text { MtChr1:21952074:21952198: } \\
+\end{array}$ & $3^{\prime}$ & 125 & -45.1 \\
\hline $\operatorname{miR} 2111 u$ & uaaucugcauccugagguuua & 21 & $413 / 23$ & MtChr7:14331003:14331117:- & $5^{\prime}$ & 115 & -50.2 \\
\hline $\operatorname{miR} 2111 v$ & uaaucugcauccugagguuua & 21 & $1192 / 41$ & MtChr7:14356392:14356499:- & $5^{\prime}$ & 108 & -48.4 \\
\hline miR2592a & gaaaaacaugaaugucgagcg & 21 & 28/1 & $\begin{array}{c}\text { MtChr1:27380857:27381050: } \\
+\end{array}$ & $3^{\prime}$ & 194 & -89.4 \\
\hline miR2592bl & uggcaaguuugaauuuaccuca & 22 & $43 / 1$ & $\begin{array}{c}\text { MtChr4:18239825:18239954: } \\
+\end{array}$ & $5^{\prime}$ & 130 & -69.3 \\
\hline miR2592bm & ggaaaacaugaaugucgggug & 21 & $918 / 294$ & MtChr5:3041012:3041204:+ & $3^{\prime}$ & 193 & -104.4 \\
\hline miR2592bn & ggaaaacaugaaugucgggug & 21 & $530 / 160$ & MtChr5:8400182:8400375:+ & $3^{\prime}$ & 194 & -111.8 \\
\hline $\operatorname{miR2619b}$ & auauguuuugauucuuuggca & 21 & $9 / 3$ & MtChr4:6335670:6335840:+ & $5^{\prime}$ & 171 & -94.7 \\
\hline $\operatorname{miR2643b}$ & uuugggaucagaaauuagaga & 21 & $361 / 11$ & MtChr5:41836449:41836558:- & $3^{\prime}$ & 110 & -38.8 \\
\hline $\operatorname{miR4414a}$ & agcugcugacucguugguuca & 21 & $663 / 45$ & $\begin{array}{c}\text { MtChr1:30518803:30518924: } \\
+\end{array}$ & $5^{\prime}$ & 122 & -50.5 \\
\hline
\end{tabular}




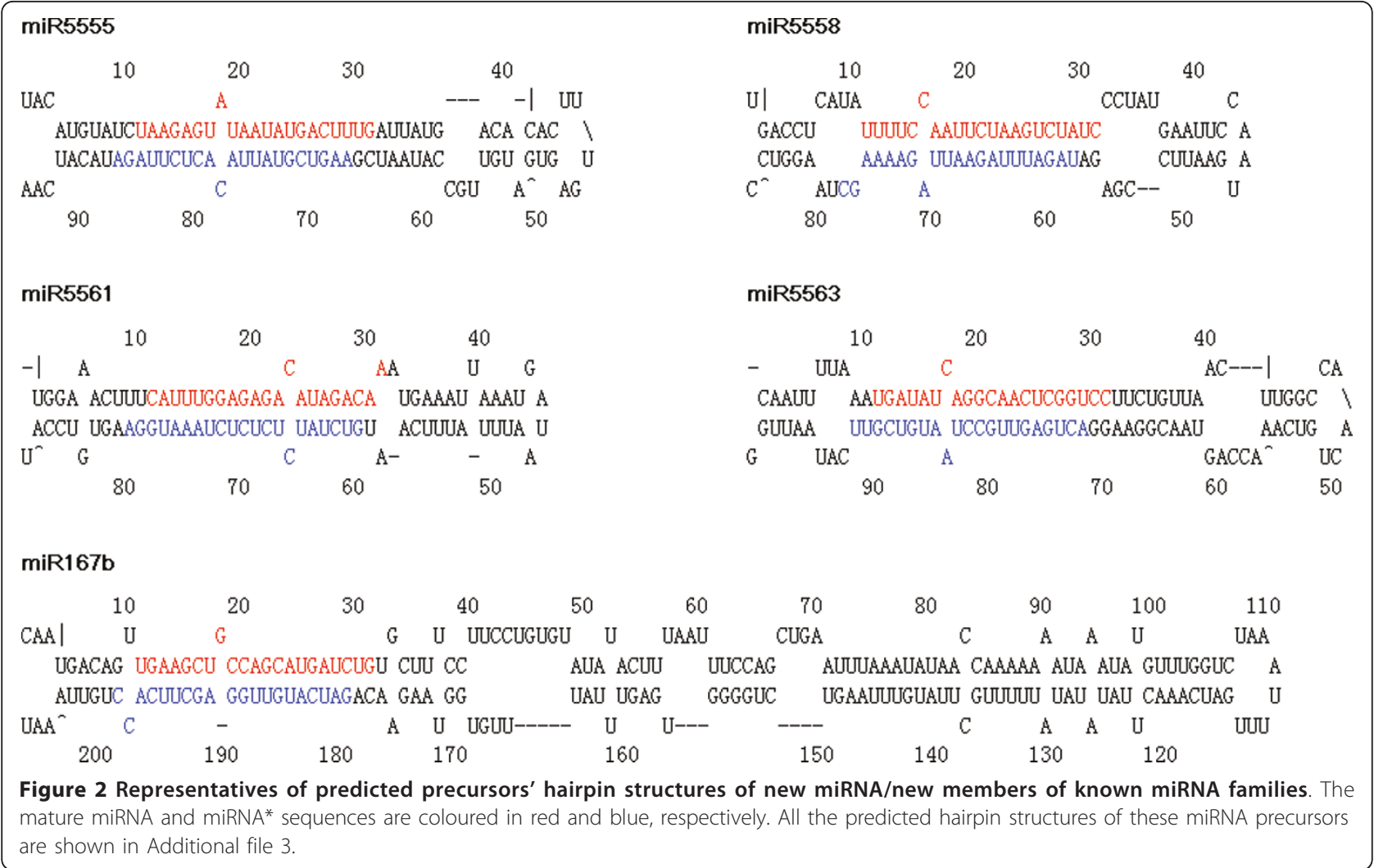

Twenty-two out of 29 new miRNAs/new members of known miRNA families had length of $21 \mathrm{nt}$, while remaining miRNAs had length of $22 \mathrm{nt}$. The majority of the miRNAs started with a 5' uridine, a hallmark of miRNAs [60]. The minimum free energy (MFE) for hairpin structure of miRNA precursors was lower than $-30 \mathrm{kcal} /$ mol (Table 2). This feature is in agreement with other reported values in the literature [61]. The length of new miRNAs' precursors ranged from 86 nt to 315 nt (Table 2). These values are comparable to those reported for $M$. truncatula in the literature $[42,51,52,62,63]$.

\section{Response of known miRNAs to drought stress}

To identify drought-responsive miRNAs, the normalized expression of miRNAs in the two libraries (control, CK and drought stress, DS) was compared. Based on the results of high-throughput sequencing, we selected those miRNAs with changes in expression levels being greater than 1.5-fold in response to drought treatment (Figure 3a and $3 \mathrm{~b}$, Additional file 4) to validate the expression patterns by real-time quantitative PCR. As shown in Figure $3 \mathrm{~b}$ and $3 c$, expression patterns of drought-responsive miRNAs from high-throughput sequencing and RT-qPCR exhibited similar results. Results from both the methods showed that 22 members in 4 miRNA families, i.e., miR399, miR2089, miR2111 and miR2118, were up-regulated in response to the drought stress. Conversely, 10 members belonging to 6 miRNA families, i.e., miR164, miR169, miR171, miR396, miR398 and miR1510, were down-regulated in response to drought stress (Figure $3 \mathrm{~b}$ and 3c). These miRNAs have been reported to be involved in diverse cellular processes in plants $[14,17,22,52]$. The known target genes of these miRNAs and their function annotations were summarized in Table 3. For those miRNAs whose targets are not known, we predicted their targets using the psRNATarget http://bioinfo3.noble.org/psRNATarget/ and the srna-tools http://srna-tools.cmp.uea.ac.uk/plant/[64], and the results are given in the Table 4 . Among these miRNAs, several miRNAs have been reported to be involved in abiotic stresses. For example, miR399 and miR2111 have been reported to be up-regulated by phosphate starvation [23,65], while miR169 with target of CCAAT Binding Factor is downregulated in response to drought stress $[14,17]$.

Response of new miRNAs/new members in known miRNA families to drought stress

We further examined whether the 29 new miRNAs/new members of known miRNA families were responsive to drought stress using the same method as the known miRNAs. The miRNAs with changes in expression levels being greater than 1.5-fold and $p$-values less than 0.05 are presented in Table 4. Results from high-throughput 

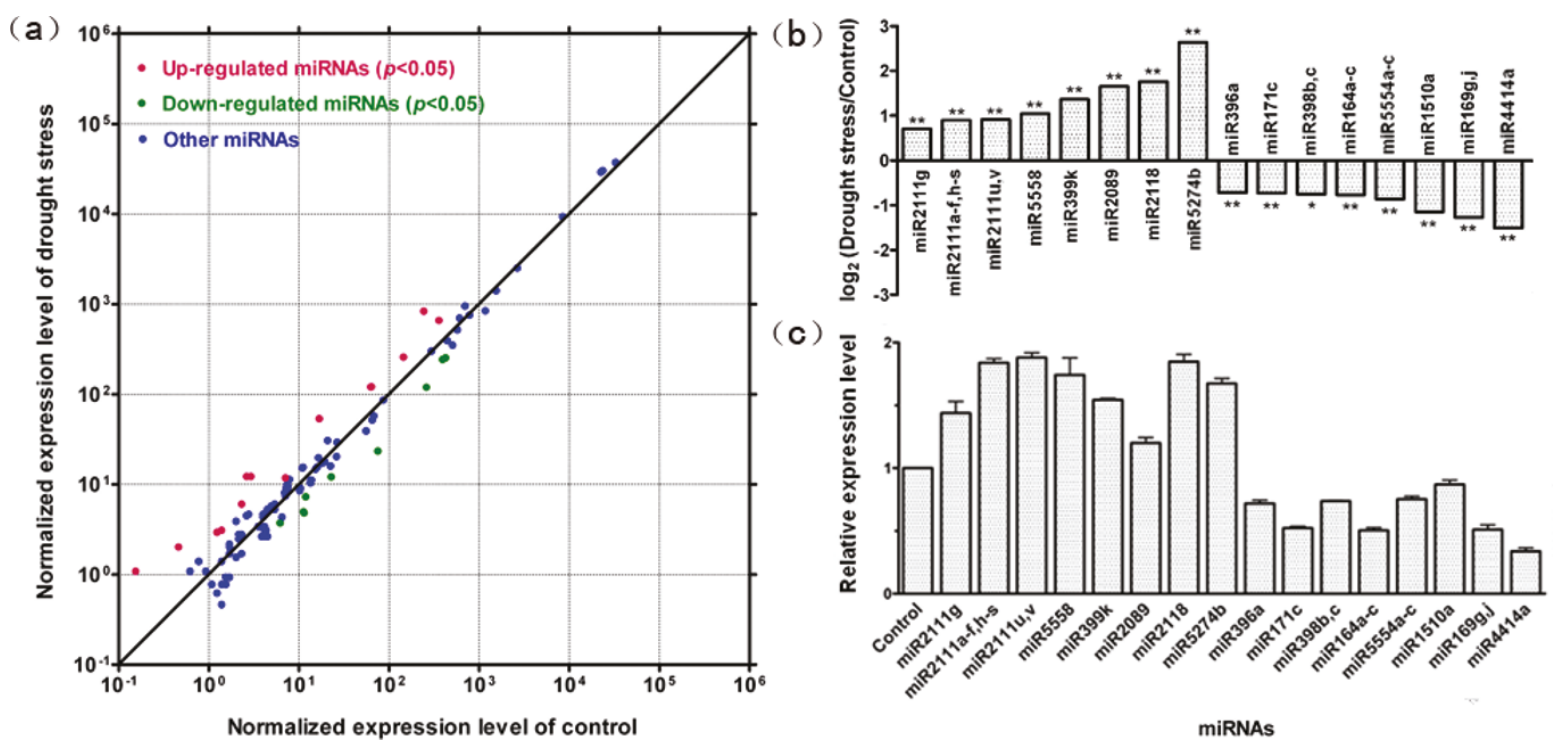

Figure 3 Differential expression analysis between control and drought stress. Data points at the upper and lower side of the slope line represent up- and down-regulated miRNAs in panel (a), respectively. The changes in miRNAs for up- and down-regulated ones are greater than 1.5-fold. Other miRNAs include those that are not responsive to drought stress and those changes induced by drought stress at $p>0.05$. Expression of control and drought stress was normalized on the basis of $1 \mathrm{M}$ reads. Differential expression of known miRNAs in response to drought stress is shown in panel (b). The positive and negative values mean miRNAs whose expression was stimulated and suppressed by drought stress, respectively. ${ }^{*}$ and ${ }^{* *}$ mean significant difference between control and drought stress at $0.01<p \leq 0.05$ and $p \leq 0.01$, respectively. The relative expression level of miRNAs measured by RT-qPCR in response to drought stress is shown in panel (c).

sequencing and RT-qPCR showed that, among the 14 new miRNAs, 5 miRNAs were responsive to drought stress with miR5274b and miR5558 being up-regulated, while miR5554a-c being down-regulated. In addition, among the 15 new members of known miRNA families, 3 miRNAs were responsive to drought stress with miR2111u, v being up-regulated and miR4414a being down-regulated (Figure 3b and 3c, Table 4).

\section{Discussion}

High-throughput sequencing of $M$. truncatula small RNAs High-throughput sequencing has been used to study miRNAs at whole genome level in several plant species, including Arabidopsis [39,40], rice [43], wheat [44], soybean [45], cotton [46], grapevine [53], Medicago [42,51,52], Brachypodium [20], tomato [41], populus [47], citrus [48], peanut [49], Porphyra [50]. However, most of the studies on high-throughput sequencing miRNAs have focused on miRNAs under non-stressed, normal growth conditions, and only a few studies have compared miRNAs under control conditions to those under conditions of abiotic stresses by high-throughput sequencing plant miRNAs [20,43,44]. For instance, Sunkar et al. (2008) reported a total of 714,202 reads in rice seedlings from three independent libraries (control, drought and salt stress libraries) [43]. Twenty-eight and 12 miRNAs in response to cold

Table 3 The known targets of drought-responsive miRNAs and their function annotations

\begin{tabular}{|c|c|c|c|c|}
\hline $\begin{array}{l}\text { miRNA } \\
\text { families }\end{array}$ & $\begin{array}{l}\text { Expression } \\
\text { pattern }\end{array}$ & Targets & Functions and responsiveness & References \\
\hline miR164 & down & $\begin{array}{l}\text { NAC domain transcription } \\
\text { factors }\end{array}$ & lateral root development & {$[9]$} \\
\hline miR169 & down & CCAAT Binding Factor (CBF) & response to drought, cold and salinity, nodule development & {$[14,15,17,18,22,33,74]$} \\
\hline miR171 & down & GRAS transcription factors & $\begin{array}{c}\text { response to drought, cold and salinity, nodule } \\
\text { morphogenesis, floral development }\end{array}$ & {$[5,18,33]$} \\
\hline miR396 & down & Growth Regulating Factor (GRF) & response to drought and salinity, cell proliferation & {$[18,52,75]$} \\
\hline miR398 & down & $\begin{array}{l}\text { Cu/Zn superoxide dismutases } \\
\text { (CSD1, CSD2) }\end{array}$ & response to oxidative stress & {$[15,27]$} \\
\hline miR399 & up & $\begin{array}{l}\text { PHO2/ubiquitin conjugating } \\
\text { enzyme }\end{array}$ & balance of phosphorus & {$[23]$} \\
\hline $\operatorname{miR} 2118$ & up & TIR-NBS-LRR domain protein & response to drought, cold, salinity, and $A B A$ & {$[51,76]$} \\
\hline
\end{tabular}


Table 4 Predicted targets of drought-responsive miRNAs

\begin{tabular}{ccc}
\hline miRNAs & $\begin{array}{c}\text { Expression } \\
\text { pattern }\end{array}$ & Predicted targets \\
\hline miR1510a & down & 1. pyruvate decarboxylase (PDC) isozyme \\
& & 2. concanavalin A-like lectin/glucanase \\
3. F-box protein & NB-ARC domain protein \\
miR2089 & up & 1. calcineurin-like phosphoesterase \\
miR2111a- & up & 2. membrane protein SAK \\
s & & 1. calcineurin-like phosphoesterase \\
family protein
\end{tabular}

stress in Brachypodium [20,44] and heat stress in wheat [44] have been identified by high-throughput sequencing, respectively. In the present study, we constructed two sRNA libraries, non-stressed, control and drought stressed in legume model plant, $M$. truncatula and identified known and new miRNAs that were responsive to drought stress.

The successful application of high-throughput sequencing technology to systemically identify plant miRNAs has greatly advanced our knowledge on the functions of miRNAs in plants in recent years. There have been several reports on the identification of miRNAs in $M$. truncatula in the literature. For instance, 26,656 and 844,110 sRNA reads were reported in two recent studies from $M$. truncatula by Jagadeeswaran et al. (2009) and Lelandais-Briere et al. (2009), respectively, with 97,028 unique sequences being obtained by the latter studies [51,52]. However, in both cases, sRNAs were sequenced using Roche 454 sequencer, and the total reads and unique sequences obtained from these studies are much less than we reported in the present study (Table 1 ). In a study by Szittya et al. (2008), the authors obtained 3,948,871 reads and $1,563,959$ unique sequences from two sRNAs libraries of $M$. truncatula using the same high-throughput sequencing technology (Illumina-Solexa) [42]. In contrast to these reported findings, we got a total of $13,683,619$ reads and $4,878,445$ unique sequences from two libraries of $M$. truncatula. Therefore, our results contain more reads than those previously reported for miRNAs in M. truncatula in the literature. Moreover, the high-throughput sequencing technology used in the present study allows us to identify the miRNAs with low abundance, thus accounting for the greater unique sequences found in our studies. In addition to the greater amounts of miRNAs sequencing data, the use of most updated miRBase also contributes to our identification of more known miRNAs in M. truncatula. The available database for $M$. truncatula contains more miRNAs than those for other plant species. For example, in the database, there are 19 miRNAs and 41 miRNAs for Brachypodium and wheat, respectively, while there are 375 miRNAs in the database for M. truncatula (miRBase 17). In addition, to the best of our knowledge, we are the first one to use the $M$. truncatula genome sequence Mt3.0 to analyze miRNAs in this species. The more genomic information in Mt3.0 than Mt2.0 may also contribute to predicting more new miRNAs in M. truncatula.

\section{Drought-responsive miRNAs}

Plant miRNAs have a strong propensity to target genes associated with development, particularly those genes encoding transcription factors and F-box proteins [25]. In the present study, we found that the known or predicted targets of miR164, miR169, miR171, miR396, miR1510 and miR5558 were either transcription factors or F-box proteins (Table 3,4). Under drought stress, an increase in root/shoot ratio was found in M. truncatula (data not shown). miR164 has been reported to regulate root development by a homeostatic mechanism to clear NAC1 mRNA, leading to down-regulation of auxin signals. It has been shown that decreases in NAC1 mRNA levels due to inducible expression of miR164, thus resulting in reduction in lateral root emergence in Arabidopsis [9]. Therefore, it is conceivable that the suppression of miR164 expression may contribute to the increase in root/shoot ratio under drought stress.

Several studies have revealed that miR169 is responsive to abiotic stresses such as drought, cold and salinity in different species $[14,15,17,22,33]$. Under drought stress, miR169 exhibited different expression patterns among different species. For example, in Arabidopsis, miR169 is down-regulated by drought stress through an ABA-dependent pathway, resulting in accumulation of NFYA5 with high affinity and sequence specificity for the CCAAT box, which is crucial for the expression of a number of drought-responsive genes [14]. In contrast to Arabidopsis, miR169g in rice is up-regulated by drought $[17,18]$. In rice, DREs (dehydration-responsive element) as the upstream of MIR169g are regulated by cold and drought stress, thus leading to up-regulation of miR169g [17]. Trindade et al. (2010) reported that the expression of miR169 in leaves of $M$. truncatula is not responsive to drought stress [15]. In contrast, our results from both the high-throughput sequencing and RT-qPCR showed that miR169 was down-regulated under drought stress in M. truncatula (Figure 3b and 3c, Additional file 4). This discrepancy may result from the difference in degree of drought stress exposed to plant materials in the two studies. For instance, in the present study, the drought 
stressed samples were collected after exposing of plants to drought for varying period (6, 8, 10 and $12 \mathrm{~d}$ after withholding water), thus our samples included plant materials suffering from a wide range of drought stress. This type of stress has been used in study of molecular response of plants to drought stress $[66,67]$. In the studies of Trindade et al. (2010), water stress was exposed by suppressing water supply with relative water content in leaves being approx. 50\% and 30\%, respectively [15]. In the present paper, the drought stressed leaves with relative water content of $87.4 \%, 78.8 \%, 75.2 \%$ and $68.3 \%$ were used for analysis of miRNAs. Therefore, our samples were mainly those suffering from mild and moderate water stress compared to those of Trindale et al. (2010). If miR169 is responsive to mild and/or early drought stress exclusively, the responsiveness of miR169 may not be detected by the more severe drought stress used by the authors [15]. In addition to withholding water supply, several studies on effect of drought on miRNAs also treated plants with PEG or mannitol for varying period $[17,33]$. It is difficult to compare the natural drought stress with PEG-induced and/or mannitol osmotic stress as the two treatments may differ in induction of water stress in terms of rapidity and severity.

Accumulation of reactive oxygen species (ROS) is a common phenomenon in response of plants to abiotic stress. The accumulated ROS damage nucleic acid, oxidize proteins and cause lipid peroxidation [68,69]. Superoxide dismutases (SODs) detoxify superoxide radicals. The targets of miR398 are two $\mathrm{Cu} / \mathrm{Zn}$ superoxide dismutases (cytosolic CSD1 and chloroplastic CSD2), and miR398 expression was reported to be down-regulated transcriptionally by oxidative stresses [27]. Oxidative stress often occurs concurrently with drought stress. In the present study, we found that miR398 was down-regulated under drought stress. This would lead to increases in activities of SODs. The drought-induced down-regulation of miR398 in $M$. truncatula is consistent with the results in maize [16], but it is contrast to the results reported by Trindale et al. (2010) [15] and Kantar et al. (2011) [34]. The differences in the expression of miR398 among different studies may results from differences in species, extent and duration of drought stress in different studies.

Plants suffering from water-deficit often display reduced uptake of mineral nutrients. In this context, miR399 negatively regulates the concentration of inorganic phosphate (Pi) by targeting PHO2, a type of E2 conjugase, and overexpression of miR399 in Pi-replete conditions represses expression of E2 conjugase, leading to an increase in $\mathrm{Pi}$ concentration in leaves in Arabidopsis [23]. Expression of miR399 is reduced under Pi-deprived conditions to facilitate accumulation of $\mathrm{Pi}$ in plants. It has been verified that miR2111 is up-regulated by Pi starvation [65]. In our study, the expression miR399 and miR2111 was similar under drought stress. These results indicate that the function of miR2111 may be as important as miR399 in regulation of nutrient acquisition.

Exposure of plants to a moderate stress induces resistance to other stresses, a phenomenon known as cross adaptation, which has been found in different combinations of stresses [70]. For example, osa-miR821 isolated from virus-infected rice tissues is also expressed in roots of salt-stressed plants, while it is not expressed in healthy, non-stressed plants [71]. Under drought stress, we found that there was up-regulation of miR2089 and miR2118, whose targets may be proteins associated with disease resistance. It is envisaged that these miRNAs may enhance the ability of drought tolerance through unknown mechanisms associated with cross adaptation in plants. Future work aiming at functional elucidation of these miRNAs is warranted by over-expressing these miRNAs in M. truncatula.

\section{Conclusions}

We obtained a total of 13,683,619 reads from two small RNA libraries of $M$. truncatula by high-throughput sequencing. Twenty-two members in 4 miRNA families and 10 members belonging to 6 miRNA families were found to be up-regulated and down-regulated in response to drought stress by both high-throughput sequencing and RT-qPCR. In addition, we also predicted 29 new miRNAs/ new members of known miRNA families, of which 8 miRNAs were responsive to drought stress by high-throughput sequencing and RT-qPCR. These findings provide valuable information for further functional characterization of miRNAs in response to abiotic stress in general and drought stress in particular in legume plants.

\section{Methods}

\section{Plant materials and drought stress treatment}

Four Medicago truncatula (cv Jemalong A17) seedlings were grown in a pot (diameter $10 \mathrm{~cm}$ ) filled with vermiculite: peat soil $(2: 1)$ under controlled conditions $\left(26^{\circ} \mathrm{C}\right.$ day/ $20^{\circ} \mathrm{C}$ night, 14 -h photoperiod, and $50 \%$ relative humidity). Drought stress was initiated by withholding water supply to 3-week-old seedlings for varying periods after seedlings were fully watered. The severely wilted leaves appeared on the 14th day after the water withholding. The soil water content was reduced from approx. $60 \%$ to $8.1 \%$ during the drought stress. Shoots under drought stress were harvested after withholding of water for 6, 8, 10 and $12 \mathrm{~d}$, and mixed averagely as drought treatment materials (DS). The relative water content for leaves collected after 6, 8, 10 and $12 \mathrm{~d}$ drought stress was $87.4 \%, 78.8 \%, 75.2 \%$ and $68.3 \%$, respectively. At the same time, shoots of $M$. truncatula seedlings grown under normal watering conditions were also harvested as control (CK). The relative water content for control leaves was $91.4 \%$. The measure method of relative water content was described by Catsky (1960) [72]. 


\section{Small RNA libraries construction for high-throughput sequencing}

To construct small RNA libraries, total RNA was extracted from shoots of control (CK) and drought stress (DS) using the Trizol (Invitrogen) according to the manufacturer's protocols. For each sample, the 18-30 nt small RNAs were ligated with 5' and 3' RNA adapter by T4 RNA ligase (TaKaRa) after they were purified by electrophoretic separation on a $15 \%$ TBE-urea denaturing PAGE gel, and at each step purified by urea PAGE gel electrophoretic separation. The RNA was subsequently transcribed to single-stranded cDNA using Superscript II reverse transcriptase (Invitrogen). Thereafter the cDNA was used as templates for double-stranded cDNA synthesis by PCR amplification using primers that anneal to adapters. The purified DNA was sequenced on a Solexa sequencer (Illumina). The raw data have been submitted to Gene Expression Omnibus (GEO, http://www.ncbi.nlm.nih.gov/ projects/geo/) and the accession number is GSE29154.

\section{Bioinformatics analysis}

After the sequence tags from Solexa sequencing went through the data cleaning by removing the low quality tags (i.e., tags less than $18 \mathrm{nt}$ and tags whose adaptors were null) and contaminants (adaptors and polyA), the length distribution and common/specific sequences between two samples were analyzed. After removing adaptor sequences of the left high-quality small RNA reads with exact matches to the adaptor sequences, reads were mapped to the M. truncatula genome sequence (Mt3.0) downloaded from the website http://www.medicagohapmap.org/downloads_genome/Mt3 using SOAP [54]. rRNAs, tRNAs, snRNAs and snoRNAs were removed from the matched sequences through BLASTn search [73] using NCBI Genebank database http://www.ncbi.nlm.nih. gov/blast/Blast.cgi/ and Rfam database http://www.sanger. ac.uk/Software/Rfam/. Mismatches were not allowed in the above two approaches. The unique sequences left were aligned with known miRNAs from miRBase 17 http:// www.mirbase.org/[55]. The potential candidate miRNAs were identified by folding the flanking genome sequence of unique small RNAs using the mfold web server [57]. Parameters were set based on the criteria for annotation plant miRNAs [58]. Target predictions were performed using the psRNATarget http://bioinfo3.noble.org/psRNATarget/ and the srna-tools http://srna-tools.cmp.uea.ac.uk/ plant/[64], through aligned with genome of $M$. truncatula and A. thaliana.

\section{Differential expression analysis of miRNAs under the drought stress}

The frequency of miRNAs of two libraries was normalized to one million by total number of miRNAs in each sample (Normalized expression = Actual miRNA count $/$ Total count of clean reads*1,000,000). miRNAs whose normalized expression of two libraries is smaller than one were removed, because their expression levels are too low. The fold change between treatment and control was calculated as: Fold-change $=\log _{2}(\mathrm{DS} / \mathrm{CK})$. The statistical analysis was performed according to Poisson distribution. The $p$-value was calculated by the following formula.

$$
\begin{aligned}
& p(x \mid \mathrm{y})=\left(\frac{N_{2}}{N_{1}}\right)^{y} \frac{(x+y) !}{x ! y !\left(1+\frac{N_{2}}{N_{1}}\right)^{(x+y+1)}} \\
& C\left(y \leq y_{\min } \mid \mathrm{x}\right)=\sum_{\gamma=0}^{\gamma \leq \gamma_{\min }} p(y \mid \mathrm{x}) \\
& D\left(y \geq y_{\max } \mid \mathrm{x}\right)=\sum_{\gamma \geq \gamma_{\max }}^{\infty} p(y \mid \mathrm{x})
\end{aligned}
$$

\section{Real-time quantitative PCR of mature miRNAs}

RT-qPCR was used to validate the results obtained from the high-throughput sequencing of miRNAs. RNA that was isolated using the Trizol (Invitrogen) as described above was reversely transcribed using One Step PrimeScript miRNA cDNA Synthesis Kit (TaKaRa). This kit adds ploy (A) to the 3' end of miRNAs and start to reversely transcribe. The reverse transcription was led by a kind of special oligo-dT ligated with known sequence at its 5' end. RT-qPCR was performed using SYBR Premix Ex Tag II (TaKaRa) and all the primers used were listed in Additional file 5. Small nuclear RNA U6 was used as internal control. Subsequently, RTqPCR was performed using Stratagene $M \times 3000 P$ instrument.

\section{Additional material}

Additional file 1: The miRNA abundance of non-conserved miRNA families in control (CK) and drought stress (DS) libraries.

Additional file 2: Potential miRNA candidates without miRNA*s in M. truncatula.

Additional file 3: The predicted hairpin structures of all the 29 new miRNAs/new members of known miRNA families' precursors.

Additional file 4: Known miRNAs in response to drought stress

Additional file 5: The primers designed for RT-qPCR.

\section{Acknowledgements}

This work was supported by the State Key Basic Research Development Program of China (2007CB106800) and Natural Science Foundation of China (No. 30821062) and State Key Laboratory of Vegetation and Environmental Change. 


\section{Author details}

IState Key Laboratory of Vegetation and Environmental Change, Institute of Botany, the Chinese Academy of Sciences, Beijing 100093, PR China.

${ }^{2}$ Graduate University of the Chinese Academy of Sciences, Beijing 100049, PR China.

\section{Authors' contributions}

TW LC WZ designed the experiments; TW LC conducted the experiments; TW LC MZ QT analyzed the data; TW WZ wrote the paper. All authors read and approved the final manuscript

Received: 1 December 2010 Accepted: 15 July 2011

Published: 15 July 2011

\section{References}

1. Jones-Rhoades MW, Bartel DP, Bartel B: MicroRNAs and their regulatory roles in plants. Annu Rev Plant Biol 2006, 57:19-53.

2. Khraiwesh B, Arif MA, Seumel Gl, Ossowski S, Weigel D, Reski R, Frank W: Transcriptional control of gene expression by microRNAs. Cell 2010, 140(1):111-122.

3. Lee $R C$, Feinbaum $R L$, Ambros V: The $C$. elegans heterochronic gene lin-4 encodes small RNAs with antisense complementarity to lin-14. Cell 1993, 75(5):843-854.

4. Bartel DP: MicroRNAs: genomics, biogenesis, mechanism, and function. Cell 2004, 116(2):281-297.

5. Llave C, Xie Z, Kasschau KD, Carrington JC: Cleavage of Scarecrow-like mRNA targets directed by a class of Arabidopsis miRNA. Science 2002, 297(5589):2053-2056.

6. Chen X: A microRNA as a translational repressor of APETALA2 in Arabidopsis flower development. Science 2004, 303(5666):2022-2025.

7. Brodersen P, Sakvarelidze-Achard L, Bruun-Rasmussen M, Dunoyer $P$, Yamamoto YY, Sieburth L, Voinnet O: Widespread translational inhibition by plant miRNAs and siRNAs. Science 2008, 320(5880):1185-1190.

8. Aukerman MJ, Sakai H: Regulation of flowering time and floral organ identity by a MicroRNA and its APETALA2-like target genes. Plant Cell 2003, 15(11):2730-2741.

9. Guo HS, Xie Q, Fei JF, Chua NH: MicroRNA directs mRNA cleavage of the transcription factor NAC1 to downregulate auxin signals for arabidopsis lateral root development. Plant Cell 2005, 17(5):1376-1386.

10. Palatnik JF, Allen E, Wu X, Schommer C, Schwab R, Carrington JC, Weigel D: Control of leaf morphogenesis by microRNAs. Nature 2003 425(6955):257-263

11. Vaucheret $H$, Vazquez F, Crete P, Bartel DP: The action of ARGONAUTE1 in the miRNA pathway and its regulation by the miRNA pathway are crucial for plant development. Genes Dev 2004, 18(10):1187-1197.

12. Mallory AC, Bartel DP, Bartel B: MicroRNA-directed regulation of Arabidopsis AUXIN RESPONSE FACTOR17 is essential for proper development and modulates expression of early auxin response genes. Plant Cell 2005, 17(5):1360-1375.

13. Wang JW, Wang L, Mao YB, Cai WJ, Xue HW, Chen XY: Control of root cap formation by MicroRNA-targeted auxin response factors in Arabidopsis. Plant Cell 2005, 17(8):2204-2216

14. Li WX, Oono Y, Zhu J, He XJ, Wu JM, lida K, Lu XY, Cui X, Jin H, Zhu JK: The Arabidopsis NFYA5 transcription factor is regulated transcriptionally and posttranscriptionally to promote drought resistance. Plant Cell 2008 20(8):2238-2251

15. Trindade I, Capitao C, Dalmay T, Fevereiro MP, Santos DM: miR398 and miR408 are up-regulated in response to water deficit in Medicago truncatula. Planta 2010, 231(3):705-716.

16. Wei LY, Zhang DF, Xiang F, Zhang ZX: Differentially Expressed miRNAs Potentially Involved in the Regulation of Defense Mechanism to Drought Stress in Maize Seedlings. Int J Plant Sci 2009, 170(8):979-989.

17. Zhao B, Liang R, Ge L, Li W, Xiao H, Lin H, Ruan K, Jin Y: Identification of drought-induced microRNAs in rice. Biochem Biophys Res Commun 2007, 354(2):585-590.

18. Zhou L, Liu Y, Liu Z, Kong D, Duan M, Luo L: Genome-wide identification and analysis of drought-responsive microRNAs in Oryza sativa. J Exp Bot 2010, 61(15):4157-4168

19. Lv DK, Bai X, Li Y, Ding XD, Ge Y, Cai H, Ji W, Wu N, Zhu YM: Profiling of cold-stress-responsive miRNAs in rice by microarrays. Gene 2010, 459(12):39-47.
20. Zhang J, Xu Y, Huan Q, Chong K: Deep sequencing of Brachypodium small RNAs at the global genome level identifies microRNAs involved in cold stress response. BMC Genomics 2009, 10:449.

21. Zhou X, Wang G, Sutoh K, Zhu JK, Zhang W: Identification of coldinducible microRNAs in plants by transcriptome analysis. Biochim Biophys Acta 2008, 1779(11):780-788.

22. Zhao B, Ge L, Liang R, Li W, Ruan K, Lin H, Jin Y: Members of miR-169 family are induced by high salinity and transiently inhibit the NF-YA transcription factor. BMC Mol Biol 2009, 10:29.

23. Bari R, Datt Pant B, Stitt M, Scheible WR: PHO2, microRNA399, and PHR1 define a phosphate-signaling pathway in plants. Plant Physiol 2006, 141(3):988-999.

24. Chiou TJ, Aung K, Lin SI, Wu CC, Chiang SF, Su CL: Regulation of phosphate homeostasis by MicroRNA in Arabidopsis. Plant Cell 2006, 18(2):412-421

25. Jones-Rhoades MW, Bartel DP: Computational identification of plant microRNAs and their targets, including a stress-induced miRNA. Mol Cell 2004, 14(6):787-799.

26. Zeng HQ, Zhu YY, Huang SQ, Yang ZM: Analysis of phosphorus-deficient responsive miRNAs and cis-elements from soybean (Glycine max L.). J Plant Physiol 2010, 167(15):1289-1297.

27. Sunkar R, Kapoor A, Zhu JK: Posttranscriptional induction of two Cu/Zn superoxide dismutase genes in Arabidopsis is mediated by downregulation of miR398 and important for oxidative stress tolerance. Plant Cell 2006, 18(8):2051-2065.

28. Zhang Z, Wei L, Zou X, Tao Y, Liu Z, Zheng Y: Submergence-responsive MicroRNAs are potentially involved in the regulation of morphological and metabolic adaptations in maize root cells. Ann Bot 2008, 102(4):509-519.

29. Jia X, Ren L, Chen QJ, Li R, Tang G: UV-B-responsive microRNAs in Populus tremula. J Plant Physio/ 2009, 166(18):2046-2057.

30. Zhou $X$, Wang G, Zhang W: UV-B responsive microRNA genes in Arabidopsis thaliana. Mol Syst Biol 2007, 3:103

31. Bazzini AA, Hopp HE, Beachy RN, Asurmendi S: Infection and coaccumulation of tobacco mosaic virus proteins alter microRNA levels, correlating with symptom and plant development. Proc Natl Acad Sci USA 2007, 104(29):12157-12162

32. He XF, Fang YY, Feng L, Guo HS: Characterization of conserved and novel microRNAs and their targets, including a TuMV-induced TIR-NBS-LRR class R gene-derived novel miRNA in Brassica. FEBS Lett 2008, 582(16):2445-2452

33. Liu HH, Tian X, Li YJ, Wu CA, Zheng CC: Microarray-based analysis of stress-regulated microRNAs in Arabidopsis thaliana. RNA 2008, 14(5):836-843

34. Kantar M, Lucas SJ, Budak H: miRNA expression patterns of Triticum dicoccoides in response to shock drought stress. Planta 2011, 233(3):471-484.

35. Benedito VA, Torres-Jerez I, Murray JD, Andriankaja A, Allen S, Kakar K, Wandrey M, Verdier J, Zuber $H$, Ott T, et al: A gene expression atlas of the model legume Medicago truncatula. Plant J 2008, 55(3):504-513.

36. Trinh $T H$, Ratet $P$, Kondorosi $E$, Durand $P$, Kamate $K$, Bauer $P$, Kondorosi $A$ : Rapid and efficient transformation of diploid Medicago truncatula and Medicago sativa ssp falcata lines improved in somatic embryogenesis. Plant Cell Rep 1998, 17(5):345-355.

37. Axtell MJ, Bartel DP: Antiquity of microRNAs and their targets in land plants. Plant Cell 2005, 17(6):1658-1673.

38. Allen E, Xie Z, Gustafson AM, Sung GH, Spatafora JW, Carrington JC: Evolution of microRNA genes by inverted duplication of target gene sequences in Arabidopsis thaliana. Nat Genet 2004, 36(12):1282-1290.

39. Rajagopalan R, Vaucheret H, Trejo J, Bartel DP: A diverse and evolutionarily fluid set of microRNAs in Arabidopsis thaliana. Genes Dev 2006, 20(24):3407-3425.

40. Fahlgren $\mathrm{N}$, Howell MD, Kasschau KD, Chapman EJ, Sullivan CM, Cumbie JS, Givan SA, Law TF, Grant SR, Dangl JL, et al: High-throughput sequencing of Arabidopsis microRNAs: evidence for frequent birth and death of MIRNA genes. PLoS One 2007, 2(2):e219.

41. Moxon S, Jing R, Szittya G, Schwach F, Rusholme Pilcher RL, Moulton V, Dalmay T: Deep sequencing of tomato short RNAs identifies microRNAs targeting genes involved in fruit ripening. Genome Res 2008, 18(10):1602-1609. 
42. Szittya G, Moxon S, Santos DM, Jing R, Fevereiro MP, Moulton V, Dalmay T: High-throughput sequencing of Medicago truncatula short RNAs identifies eight new miRNA families. BMC Genomics 2008, 9:593.

43. Sunkar R, Zhou X, Zheng Y, Zhang W, Zhu JK: Identification of novel and candidate miRNAs in rice by high throughput sequencing. BMC Plant Bio 2008, 8:25

44. Xin $M$, Wang $Y$, Yao $Y$, Xie C, Peng $H, N i Z$, Sun Q: Diverse set of microRNAs are responsive to powdery mildew infection and heat stress in wheat (Triticum aestivum L.). BMC Plant Biol 2010, 10:123.

45. Subramanian S, Fu Y, Sunkar R, Barbazuk WB, Zhu JK, Yu O: Novel and nodulation-regulated microRNAs in soybean roots. BMC Genomics 2008, 9:160

46. Kwak PB, Wang QQ, Chen XS, Qiu CX, Yang ZM: Enrichment of a set of microRNAs during the cotton fiber development. BMC Genomics 2009, 10:457.

47. Barakat A, Wall PK, Diloreto S, Depamphilis CW, Carlson JE: Conservation and divergence of microRNAs in Populus. BMC Genomics 2007, 8:481.

48. Song C, Wang C, Zhang C, Korir NK, Yu H, Ma Z, Fang J: Deep sequencing discovery of novel and conserved microRNAs in trifoliate orange (Citrus trifoliata). BMC Genomics 2010, 11:431.

49. Zhao CZ, Xia H, Frazier TP, Yao YY, Bi YP, Li AQ, Li MJ, Li CS, Zhang BH, Wang $X J$ : Deep sequencing identifies novel and conserved microRNAs in peanuts (Arachis hypogaea L.). BMC Plant Biol 2010, 10:3.

50. Liang C, Zhang X, Zou J, Xu D, Su F, Ye N: Identification of miRNA from Porphyra yezoensis by high-throughput sequencing and bioinformatics analysis. PLoS One 2010, 5(5):e10698.

51. Jagadeeswaran G, Zheng Y, Li YF, Shukla LI, Matts J, Hoyt P, Macmil SL, Wiley GB, Roe BA, Zhang W, et al: Cloning and characterization of small RNAs from Medicago truncatula reveals four novel legume-specific microRNA families. New Phytol 2009, 184(1):85-98

52. Lelandais-Briere C, Naya L, Sallet E, Calenge F, Frugier F, Hartmann C, Gouzy J, Crespi M: Genome-wide Medicago truncatula small RNA analysis revealed novel microRNAs and isoforms differentially regulated in roots and nodules. Plant Cell 2009, 21(9):2780-2796.

53. Pantaleo V, Szittya G, Moxon S, Miozzi L, Moulton V, Dalmay T, Burgyan J: Identification of grapevine microRNAs and their targets using highthroughput sequencing and degradome analysis. Plant J 2010, 62(6):960-976.

54. Li R, Li Y, Kristiansen K, Wang J: SOAP: short oligonucleotide alignment program. Bioinformatics 2008, 24(5):713-714.

55. Griffiths-Jones S, Saini HK, van Dongen S, Enright AJ: miRBase: tools for microRNA genomics. Nucleic Acids Res 2008, 36 Database: D154-158.

56. Ambros V, Bartel B, Bartel DP, Burge CB, Carrington JC, Chen X, Dreyfuss G, Eddy SR, Griffiths-Jones S, Marshall $M$, et al: A uniform system for microRNA annotation. RNA 2003, 9(3):277-279.

57. Zuker M: Mfold web server for nucleic acid folding and hybridization prediction. Nucleic Acids Res 2003, 31(13):3406-3415.

58. Meyers BC, Axtell MJ, Bartel B, Bartel DP, Baulcombe D, Bowman JL, Cao X, Carrington JC, Chen $X$, Green PJ, et al: Criteria for annotation of plant MicroRNAs. Plant Cell 2008, 20(12):3186-3190.

59. Wu L, Zhang Q, Zhou H, Ni F, Wu X, Qi Y: Rice MicroRNA effector complexes and targets. Plant Cell 2009, 21(11):3421-3435.

60. Yao Y, Guo G, Ni Z, Sunkar R, Du J, Zhu JK, Sun Q: Cloning and characterization of microRNAs from wheat (Triticum aestivum L.). Genome Biol 2007, 8(6):R96

61. Bonnet E, Wuyts J, Rouze P, Van de Peer Y: Evidence that microRNA precursors, unlike other non-coding RNAs, have lower folding free energies than random sequences. Bioinformatics 2004, 20(17):2911-2917.

62. Wen J, Frickey T, Weiller GF: Computational prediction of candidate miRNAs and their targets from Medicago truncatula non-protein-coding transcripts. In Silico Biol 2008, 8(3-4):291-306.

63. Zhou ZS, Huang SQ, Yang ZM: Bioinformatic identification and expression analysis of new microRNAs from Medicago truncatula. Biochem Biophys Res Commun 2008, 374(3):538-542.

64. Moxon S, Schwach F, Dalmay T, Maclean D, Studholme DJ, Moulton V: A toolkit for analysing large-scale plant small RNA datasets. Bioinformatics 2008, 24(19):2252-2253.

65. Pant BD, Musialak-Lange M, Nuc P, May P, Buhtz A, Kehr J, Walther D, Scheible WR: Identification of nutrient-responsive Arabidopsis and rapeseed microRNAs by comprehensive real-time polymerase chain reaction profiling and small RNA sequencing. Plant Physiol 2009, 150(3):1541-1555.

66. Zheng J, Zhao J, Tao Y, Wang J, Liu Y, Fu J, Jin Y, Gao P, Zhang J, Bai Y, et al: Isolation and analysis of water stress induced genes in maize seedlings by subtractive PCR and cDNA macroarray. Plant Mol Biol 2004 55(6):807-823

67. Gazendam I, Oelofse D: Isolation of cowpea genes conferring drought tolerance: Construction of a cDNA drought expression library. Water SA 2007, 33(3):387-391.

68. Apel K, Hirt H: Reactive oxygen species: metabolism, oxidative stress, and signal transduction. Annu Rev Plant Biol 2004, 55:373-399.

69. Foyer $\mathrm{CH}$, Descourvieres $\mathrm{P}$, Kunert $\mathrm{KJ}$ : Protection against oxygen radicals: An important defense mechanism studied in transgenic plants. Plant Cell Environ 1994, 17(5):507-523.

70. Sabehat A, Weiss D, Lurie S: Heat-shock proteins and cross-tolerance in plants. Physiol Plant 1998, 103(3):437-441.

71. Sanan-Mishra N, Kumar V, Sopory SK, Mukherjee SK: Cloning and validation of novel miRNA from basmati rice indicates cross talk between abiotic and biotic stresses. Mol Genet Genomics 2009, 282(5):463-474.

72. Catsky J: Determination of water deficit in discs cut out from leaf blades. Biol Plant 1960, 2:76-77.

73. Altschul SF, Gish W, Miller W, Myers EW, Lipman DJ: Basic local alignment search tool. J Mol Biol 1990, 215(3):403-410.

74. Combier JP, Frugier F, de Billy F, Boualem A, El-Yahyaoui F, Moreau S, Vernie T, Ott T, Gamas P, Crespi M, et al: MtHAP2-1 is a key transcriptional regulator of symbiotic nodule development regulated by microRNA169 in Medicago truncatula. Genes Dev 2006, 20(22):3084-3088.

75. Rodriguez RE, Mecchia MA, Debernardi JM, Schommer C, Weigel D, Palatnik JF: Control of cell proliferation in Arabidopsis thaliana by microRNA miR396. Development 2010, 137(1):103-112.

76. Arenas-Huertero C, Perez B, Rabanal F, Blanco-Melo D, De la Rosa C, Estrada-Navarrete G, Sanchez F, Covarrubias AA, Reyes JL: Conserved and novel miRNAs in the legume Phaseolus vulgaris in response to stress. Plant Mol Biol 2009, 70(4):385-401

doi:10.1186/1471-2164-12-367

Cite this article as: Wang et al:: Identification of drought-responsive microRNAs in Medicago truncatula by genome-wide high-throughput sequencing. BMC Genomics 2011 12:367.

\section{Submit your next manuscript to BioMed Central and take full advantage of:}

- Convenient online submission

- Thorough peer review

- No space constraints or color figure charges

- Immediate publication on acceptance

- Inclusion in PubMed, CAS, Scopus and Google Scholar

- Research which is freely available for redistribution

Submit your manuscript at www.biomedcentral.com/submit
C Biomed Central 